\title{
Hankel 算子乘积的两个问题
}

\author{
丁宣浩 ${ }^{1}$, 桑元琦 $2 *$
}

1. 重庆工商大学数学与统计学院, 重庆市经济社会应用统计重点实验室, 重庆 401331;

2. 复旦大学数学科学学院, 上海 200433

E-mail: xuanhaod@qq.com,yuanqisang@fudan.edu.cn

收稿日期: 2019-01-09; 接受日期: 2020-03-12；网络出版日期: 2020-08-27；＊通信作者

国家自然科学基金 (批准号: 11871122) 和重庆市自然科学基金 (批准号: cstc2018jcyjAX0595) 资助项目

摘要 设 $f 、 u$ 和 $g$ 是单位圆周 Hardy 空间 $H^{2}$ 中的函数, $h$ 是单位圆周上平方可积的函数, $H_{\bar{f}} 、 H_{\bar{u}} 、 H_{\bar{g}}$ 和 $H_{h}$ 都是从单位圆周 Hardy 空间 $H^{2}$ 到其正交补空间 $\left(H^{2}\right)^{\perp}$ 上有界的 Hankel 算子. 本文得到 了 3 个 Hankel 算子的乘积等于一个 Hankel 算子 (即 $H_{\bar{f}} H_{\bar{u}}^{*} H_{\bar{g}}=H_{h}$ ) 成立的充分必要条件, 以及 $H_{\bar{f}} H_{\bar{u}}^{*} H_{\bar{g}}=H_{\bar{g}} H_{\bar{u}}^{*} H_{\bar{f}}$ 成立的充分必要条件.

关键词 Hankel 算子 Hardy 空间 乘积

MSC (2020) 主题分类 $47 \mathrm{~B} 35$

\section{1 引言}

本文中, $\mathbb{D}$ 和 $\mathbb{T}$ 分别表示复平面中的开单位圆盘和单位圆周. $d \sigma(z)$ 是 $\mathbb{T}$ 上的正规化的 Haar 测 度. $L^{2}=L^{2}(\mathbb{T}, d \sigma)$ 为 $\mathbb{T}$ 上的平方可积函数全体, $L^{\infty}$ 为 $\mathbb{T}$ 上的本性有界函数全体. $H^{2}$ 表示 Hardy 空 间, 是 $L^{2}$ 的真闭子空间, 其定义为

$$
H^{2}=\left\{f \in L^{2}: f=\sum_{n=0}^{\infty} \hat{f}(n) z^{n}, \sum_{n=0}^{\infty}|\hat{f}(n)|^{2}<\infty\right\}
$$

利用调和扩张, $H^{2}$ 中的函数在 $\mathbb{D}$ 上解析, 且 $H^{2}$ 中的任意元素 $f$ 的范数为

$$
\|f\|=\left(\sum_{n=0}^{\infty}|\hat{f}(n)|^{2}\right)^{1 / 2},
$$

其中 $f=\sum_{n=0}^{\infty} \hat{f}(n) z^{n}$ 是 $f$ 在 $H^{2}$ 中标准正交基 $\left\{z^{n}: n=0,1,2, \ldots\right\}$ 下的 Fourier 展开式, 或解析函 数 $f$ 在 $\mathbb{D}$ 内的 Taylor 展式. 
设 $P$ 是从 $L^{2}$ 到 $H^{2}$ 上的正交投影, 记 $P_{-}=I-P$ 为从 $L^{2}$ 到 $\left(H^{2}\right)^{\perp}$ 上的正交投影. 对于 $\varphi \in L^{\infty}$, Toeplitz 算子 $T_{\varphi}$ 定义为

$$
T_{\varphi} x=P \varphi x, \quad \forall x \in H^{2} .
$$

Hankel 算子 $H_{\varphi}$ 定义为

$$
H_{\varphi} x=P_{-} \varphi x, \quad \forall x \in H^{2} .
$$

对偶 Toeplitz 算子 $S_{\varphi}$ 定义为

$$
S_{\varphi} y=P_{-} \varphi y, \quad \forall y \in\left(H^{2}\right)^{\perp}
$$

Hankel 算子的伴随 $H_{\bar{\varphi}}^{*}$ 定义为

$$
H_{\bar{\varphi}}^{*} y=P \varphi y, \quad \forall y \in\left(H^{2}\right)^{\perp} .
$$

$L^{2}$ 上的乘法算子 $M_{f}$ 在空间分解 $L^{2}=H^{2} \oplus\left(H^{2}\right)^{\perp}$ 下可以表示为如下的 $2 \times 2$ 算子矩阵:

$$
M_{f}=\left(\begin{array}{cc}
T_{f} & H_{\frac{*}{f}}^{*} \\
H_{f} & S_{f}
\end{array}\right) .
$$

由 $M_{f} M_{g}=M_{f g}$, 可得

$$
\begin{aligned}
& T_{f g}=T_{f} T_{g}+H_{\bar{f}}^{*} H_{g}, \\
& H_{f g}=H_{f} T_{g}+S_{f} H_{g}, \\
& H_{\overline{f g}}^{*}=T_{f} H_{\bar{g}}^{*}+H_{\bar{f}}^{*} S_{g}, \\
& S_{f g}=S_{f} S_{g}+H_{f} H_{\bar{g}}^{*} .
\end{aligned}
$$

若 $\varphi \in H^{2}$, 则 $H_{\varphi}=0$. 这样 (1.3) 和 (1.4) 可以推出

$$
H_{\varphi \psi}=S_{\varphi} H_{\psi}=H_{\psi} T_{\varphi}, \quad \varphi \in H^{2} .
$$

1997 年, Xia 和 Zheng ${ }^{[1]}$ 讨论了 3 个 Hankel 算子乘积为 0 和为紧算子的条件, 时隔多年数学家 们发现这些结果在讨论截断 Toeplitz 算子时非常有用. 设 $\theta$ 是一个非常数的内函数, $K_{\theta}^{2}=H^{2} \ominus \theta H^{2}$ 为模型空间, $P_{\theta}$ 表示从 $L^{2}$ 到 $K_{\theta}^{2}$ 上的正交投影. 2007 年, Sarason ${ }^{[2]}$ 引入了截断 Toeplitz 算子, 定义 如下: 对于 $\varphi \in L^{\infty}$,

$$
A_{\varphi} x=P_{\theta} \varphi x, \quad \forall x \in K_{\theta}^{2} .
$$

随后, 数学家们注意到截断 Toeplitz 算子与 3 个 Hankel 算子的乘积有如下关系 (参见文献 [3]):

$$
\left(\begin{array}{cc}
A_{\varphi} M_{\theta} & 0 \\
0 & 0
\end{array}\right)=H_{\bar{\theta}}^{*} H_{\bar{\theta} \varphi} H_{\bar{\theta}}^{*}:\left(H^{2}\right)^{\perp}=\bar{\theta} K_{\theta}^{2} \oplus \bar{\theta}\left(H^{2}\right)^{\perp} \rightarrow H^{2}=K_{\theta}^{2} \oplus \theta H^{2}
$$


Ma 和 Zheng ${ }^{[4]}$ 给出了截断 Toeplitz 算子为紧算子的充要条件, 其主要的方法是利用上面的矩阵表示 和 3 个 Hankel 算子乘积为紧的条件. 而我们在讨论两个对偶截断 Toeplitz 算子的乘积为一个对偶截 断 Toeplitz 算子时, 也遇到了 3 个 Hankel 算子乘积为 0 的刻画 (参见文献 [5]). 由此看来, 讨论 3 个 Hankel 算子的乘积的性质是非常有意义的. 由于符号为解析函数的 Hankel 算子为 0 , 所以只需考虑 符号为共轭解析函数的 Hankel 算子. 本文主要讨论关于有界 Hankel 算子的两个问题:

问题 1.1 设 $f 、 g$ 和 $u$ 是 $H^{2}$ 中的 3 个非常值函数, $h \in L^{2}$, 什么条件下算子等式

$$
H_{\bar{f}} H_{\bar{u}}^{*} H_{\bar{g}}=H_{h}
$$

成立?

问题 1.2 设 $f 、 g$ 和 $u$ 是 $H^{2}$ 中的 3 个非常值函数, 什么条件下算子等式

$$
H_{\bar{f}} H_{\bar{u}}^{*} H_{\bar{g}}=H_{\bar{g}} H_{\bar{u}}^{*} H_{\bar{f}}
$$

成立?

本文完全回答了这两个问题. 主要结果如下:

定理 1.1 设 $f 、 g$ 和 $u$ 是属于 $H^{2}$ 的 3 个非常值函数, $h \in L^{2}$, 则 $H_{\bar{f}} H_{\bar{u}}^{*} H_{\bar{g}}=H_{h}$ 成立的充分 必要条件是存在常数 $\lambda$, 使得 $(u-\lambda) \bar{f} 、(u-\lambda) \bar{g}$ 和 $(u-\lambda) \bar{f} \bar{g}-h$ 都属于 $H^{2}$.

定理 1.2 设 $f 、 g$ 和 $u$ 是属于 $H^{2}$ 的 3 个非常值函数, 则 $H_{\bar{f}} H_{\bar{u}}^{*} H_{\bar{g}}=H_{\bar{g}} H_{\bar{u}}^{*} H_{\bar{f}}$ 成立的充分必 要条件是下列条件之一成立:

(1) 存在常数 $\lambda$, 使得 $(u-\lambda) \bar{f}$ 和 $(u-\lambda) \bar{g}$ 都属于 $H^{2}$;

(2) $f$ 和 $g$ 的非平凡线性组合是常数.

\section{3 个 Hankel 算子的乘积等于一个 Hankel 算子}

1999 年, Yoshino ${ }^{[6]}$ 给出了两个 Hankel 算子的乘积等于一个 Hankel 算子的条件. 这是我们研究 问题 1.1 的另外一个重要的背景. Yoshino 定义的 Hankel 算子与本文的定义有所不同, 具体定义如下:

$$
\hat{H}_{f} h=P(U f h), \quad h \in H^{2},
$$

其中 $U h(z)=\bar{z} h(\bar{z})$ 是 $L^{2}$ 上的酉算子. 这种 Hankel 算子与本文的 Hankel 算子可以通过算子 $U$ 相互转 化, 事实上, 两种 Hankel 算子的基本性质是一致的. 因为 Hankel 算子不可能是恒等算子, 所以问题 1.1 不能约化为 Yoshino 研究的情形. 因此研究 3 个 Hankel 算子的乘积等于一个 Hankel 算子的条件就 变成了一件非常有意义的事情.

在 Toeplitz 算子与 Hankel 算子的代数运算中有一个非常有趣的现象, 即根据两个算子乘积的结 果很难推导出两个以上乘积的情况. 例如, 著名的 Brown-Halmos 定理 ${ }^{[7]}$ 给出了单位圆盘 Hardy 空间 上两个 Toeplitz 算子的乘积等于一个 Toeplitz 算子的充分必要条件. 但是, 至今不知道 3 个 Toeplitz 算子的乘积等于一个 Toeplitz 算子的条件, 当然任意有限个 Toeplitz 算子的乘积等于一个 Toeplitz 算子的充分必要条件是一个更加困难的公开问题. Ahern ${ }^{[8] 、 A h e r n ~}$ 和 Čučković ${ }^{[9]}$ 给出了单位圆盘 Bergman 空间上具有有界调和函数符号的两个 Toeplitz 算子的乘积等于一个 Toeplitz 算子的条件, 但 是刻画 3 个具有有界调和函数符号的 Toeplitz 算子的乘积等于一个 Toeplitz 算子的条件仍然是一个 没有解决的困难问题. 本节将给出 3 个 Hankel 算子的乘积等于一个 Hankel 算子的条件, 从而完全解 决问题 1.1 . 
定理 2.1 设 $f 、 g$ 和 $u$ 是属于 $H^{2}$ 的 3 个非常值函数, $h \in L^{2}$, 则 $H_{\bar{f}} H_{\bar{u}}^{*} H_{\bar{g}}=H_{h}$ 成立的充分 必要条件是存在常数 $\lambda$, 使得 $(u-\lambda) \bar{f} 、(u-\lambda) \bar{g}$ 和 $(u-\lambda) \bar{f} \bar{g}-h$ 都属于 $H^{2}$.

证明 先证充分性. 假设存在常数 $\lambda$, 使得 $(u-\lambda) \bar{f} 、(u-\lambda) \bar{g}$ 和 $(u-\lambda) \bar{f} \bar{g}-h$ 都属于 $H^{2}$. 因为 $H_{\bar{u}}^{*}=H_{\bar{u}-\bar{\lambda}}^{*}$ 成立, 所以, $H_{\bar{f}} H_{\bar{u}}^{*} H_{\bar{g}}=H_{h}$ 成立等价于

$$
H_{\bar{f}} H_{\bar{u}-\bar{\lambda}}^{*} H_{\bar{g}}=H_{h}
$$

成立. 对于任意 $z \in \mathbb{D}, K_{z}(\zeta)=\frac{1}{1-\bar{z} \zeta}$ 是 $H^{2}$ 的再生核. 根据假设, 我们将 (2.1) 两边作用在 $K_{z}$ 上可得

$$
\begin{aligned}
H_{\bar{f}} H_{\bar{u}-\bar{\lambda}}^{*} H_{\bar{g}} K_{z} & =H_{\bar{f}} H_{\bar{u}-\bar{\lambda}}^{*}(I-P) \bar{g} K_{z} \\
& =H_{\bar{f}} H_{\bar{u}-\bar{\lambda}}^{*}(\bar{g}-\bar{g}(z)) K_{z} \\
& =H_{\bar{f}} P(u-\lambda)(\bar{g}-\bar{g}(z)) K_{z} \\
& =H_{\bar{f}}\left((u-\lambda)(\bar{g}-\bar{g}(z)) K_{z}\right) \\
& =(I-P)(u-\lambda) \bar{f} \bar{g} K_{z}-\bar{g}(z)(I-P) \bar{f}(u-\lambda) K_{z} \\
& =(I-P)(u-\lambda) \bar{f} \bar{g} K_{z} \\
& =(I-P)((u-\lambda) \bar{f} \bar{g}-h) K_{z}+(I-P) h K_{z} \\
& =H_{h} K_{z} .
\end{aligned}
$$

从而, 对于任意的 $z \in \mathbb{D}, H_{\bar{f}} H_{\bar{u}}^{*} H_{\bar{g}} K_{z}=H_{h} K_{z}$ 成立. 由于 $\left\{K_{z}: z \in \mathbb{D}\right\}$ 的有限线性组合全体在 $H^{2}$ 中 稠密, 故

$$
H_{\bar{f}} H_{\bar{u}}^{*} H_{\bar{g}}=H_{h}
$$

下证必要性. 假设 $H_{\bar{f}} H_{\bar{u}}^{*} H_{\bar{g}}=H_{h}$ 成立. 由 (1.1) 可知, $H_{\bar{u}}^{*} H_{\bar{g}}=T_{u \bar{g}}-T_{u} T_{\bar{g}}$. 故

$$
\begin{aligned}
H_{\bar{f}} H_{\bar{u}}^{*} H_{\bar{g}} & =H_{\bar{f}}\left(T_{u \bar{g}}-T_{u} T_{\bar{g}}\right) \\
& =H_{\bar{f}} T_{u \bar{g}}-H_{\bar{f}} T_{u} T_{\bar{g}} \\
& =H_{\bar{f}} T_{u \bar{g}}-H_{u \bar{f}} T_{\bar{g}} .
\end{aligned}
$$

这样 $H_{\bar{f}} H_{\bar{u}}^{*} H_{\bar{g}}=H_{h}$ 变成了

$$
H_{\bar{f}} T_{u \bar{g}}-H_{u \bar{f}} T_{\bar{g}}=H_{h}
$$

又因为 $I=T_{z} T_{\bar{z}}+1 \otimes 1$, 其中 $1 \otimes 1$ 为秩一算子, 满足 $(1 \otimes 1) x=\langle x, 1\rangle 1, \forall x \in H^{2}$. 从而, $(2.2)$ 变为

$$
\begin{aligned}
& H_{\bar{f}}\left(T_{z} T_{\bar{z}}+1 \otimes 1\right) T_{u \bar{g}}-H_{u \bar{f}}\left(T_{z} T_{\bar{z}}+1 \otimes 1\right) T_{\bar{g}}=H_{h}, \\
& H_{\bar{f}}(1 \otimes 1) T_{u \bar{g}}-H_{u \bar{f}}(1 \otimes 1) T_{\bar{g}}=H_{h}-\left(H_{\bar{f}} T_{z} T_{\bar{z}} T_{u \bar{g}}-H_{u \bar{f}} T_{z} T_{\bar{z}} T_{\bar{g}}\right), \\
& H_{\bar{f}}(1 \otimes 1) T_{u \bar{g}}-H_{u \bar{f}}(1 \otimes 1) T_{\bar{g}}=H_{h}-S_{z}\left(H_{\bar{f}} T_{u \bar{z} \bar{g}}-H_{u \bar{f}} T_{\bar{z} \bar{g}}\right) .
\end{aligned}
$$

在上式两边同时复合算子 $T_{z}$, 可得

$$
\begin{aligned}
\left(H_{\bar{f}}(1 \otimes 1) T_{u \bar{g}}-H_{u \bar{f}}(1 \otimes 1) T_{\bar{g}}\right) T_{z} & =H_{h} T_{z}-S_{z}\left(H_{\bar{f}} T_{u \bar{g}}-H_{u \bar{f}} T_{\bar{g}}\right) \\
& =H_{h} T_{z}-S_{z} H_{h}
\end{aligned}
$$




$$
=H_{h z}-H_{h z}=0
$$

因此,

$$
H_{\bar{f}}(1 \otimes 1) T_{u \bar{g}} T_{z}=H_{u \bar{f}}(1 \otimes 1) T_{\bar{g}} T_{z} .
$$

把上式中每一项具体写出来, 可得

$$
P_{-}(\bar{f}) \otimes P(\bar{z} \bar{u} g)=P_{-}(u \bar{f}) \otimes P(\bar{z} g) .
$$

下面分两种情形讨论上面的算子方程.

情形 $1 \quad P_{-}(\bar{f}) \otimes P(\bar{z} \bar{u} g)$ 和 $P_{-}(u \bar{f}) \otimes P(\bar{z} g)$ 都是零算子. 因为 $f$ 和 $g$ 不是常值函数, 所以 有 $P_{-}(\bar{f}) \neq 0$ 和 $P(\bar{z} g) \neq 0$. 从而 $P(\bar{z} \bar{u} g)=0$ 且 $P_{-}(u \bar{f})=0$. 因此, $\bar{z} \bar{u} g \in\left(H^{2}\right)^{\perp}$ 且 $u \bar{f} \in H^{2}$. $\bar{z} \bar{u} g \in\left(H^{2}\right)^{\perp}$ 可以推出 $\bar{u} g \in z\left(H^{2}\right)^{\perp}=\overline{H^{2}}$, 则 $u \bar{g} \in H^{2}$. 显见

$$
H_{\bar{f}} H_{\bar{u}}^{*} H_{\bar{g}} 1=H_{h} 1 .
$$

计算 $(2.4)$ 左边可得

$$
\begin{aligned}
H_{\bar{f}} H_{\bar{u}}^{*} H_{\bar{g}} 1 & =H_{\bar{f}} H_{\bar{u}}^{*} P_{-} \bar{g} \\
& =H_{\bar{f}} H_{\bar{u}}^{*}[\bar{g}-\bar{g}(0)] \\
& =H_{\bar{f}} P u[\bar{g}-\bar{g}(0)] \\
& =P_{-} \bar{f} u[\bar{g}-\bar{g}(0)] \\
& =P_{-} u \bar{f} \bar{g}-\bar{g}(0) P_{-}(u \bar{f}) \\
& =P_{-} u \bar{f} \bar{g} .
\end{aligned}
$$

计算 $(2.4)$ 右边可得

$$
H_{h} 1=P_{-} h \text {. }
$$

因此,

$$
P_{-}(u \bar{f} \bar{g}-h)=0 \quad \Rightarrow \quad u \bar{f} \bar{g}-h \in H^{2} .
$$

情形 2 若 $P_{-}(\bar{f}) \otimes P(\bar{z} \bar{u} g)$ 和 $P_{-}(u \bar{f}) \otimes P(\bar{z} g)$ 都不是零算子, 则存在 $\mathbb{D}$ 中的一点 $z_{0}$, 使得

$$
\left\langle P(\bar{z} \bar{u} g), K_{z_{0}}\right\rangle=P(\bar{z} \bar{u} g)\left(z_{0}\right), \quad\left\langle P(\bar{z} g), K_{z_{0}}\right\rangle=P(\bar{z} g)\left(z_{0}\right)
$$

都是非零常数. 由假设可知,

$$
P_{-}(\bar{f}) \otimes P(\bar{z} \bar{u} g) K_{z_{0}}=P_{-}(u \bar{f}) \otimes P(\bar{z} g) K_{z_{0}} .
$$

计算上式可得

$$
\left\langle K_{z_{0}}, P(\bar{z} \bar{u} g)\right\rangle P_{-}(\bar{f})=\left\langle K_{z_{0}}, P(\bar{z} g)\right\rangle P_{-}(u \bar{f}) .
$$

记 $\lambda=\overline{\left(\frac{P(\bar{z} \bar{g} g)\left(z_{0}\right)}{P(\bar{z} g)\left(z_{0}\right)}\right)} \neq 0$ 使得

$$
P_{-}(u \bar{f})=\lambda P_{-}(\bar{f}) .
$$


因此,

$$
P_{-}(u \bar{f}-\lambda \bar{f})=0 \quad \Rightarrow \quad(u-\lambda) \bar{f} \in H^{2} .
$$

将 (2.5) 代入 (2.3) 可得

$$
P_{-}(\bar{f}) \otimes P(\bar{z} \bar{u} g)=\lambda P_{-}(\bar{f}) \otimes P(\bar{z} g)
$$

化简上式可得

$$
P_{-}(\bar{f}) \otimes(P(\bar{z} \bar{u} g)-\bar{\lambda} P(\bar{z} g))=0 .
$$

故

$$
\begin{aligned}
P(\bar{z}(\bar{u} g-\bar{\lambda}) g)=0 & \Rightarrow \bar{z}(\bar{u} g-\bar{\lambda}) g \in\left(H^{2}\right)^{\perp} \\
& \Rightarrow(\bar{u}-\bar{\lambda}) g \in z\left(H^{2}\right)^{\perp}=\overline{H^{2}} \\
& \Rightarrow(u-\lambda) \bar{g} \in H^{2} .
\end{aligned}
$$

由假设可得

$$
H_{\bar{f}} H_{\bar{u}-\bar{\lambda}}^{*} H_{\bar{g}} 1=H_{h} 1 .
$$

将上式展开可得

$$
\begin{aligned}
H_{\bar{f}} H_{\bar{u}-\bar{\lambda}}^{*} H_{\bar{g}} 1 & =H_{\bar{f}} P(u-\lambda)(\bar{g}-\bar{g}(0)) \\
& =P_{-} \bar{f}(u-\lambda)(\bar{g}-\bar{g}(0)) \\
& =P_{-}(u-\lambda) \bar{f} \bar{g}=P_{-} h .
\end{aligned}
$$

故

$$
(u-\lambda) \bar{f} \bar{g}-h \in H^{2} .
$$

证毕.

推论 2.1 设 $f_{1}, f_{2}, f_{3} \in L^{\infty}$, 则 $H_{f_{1}} H_{f_{2}}^{*} H_{f_{3}}=0$ 成立当且仅当存在常数 $\lambda$, 使得 $\left(f_{2-}^{-}-\lambda\right) f_{1-}$ 、 $\left(f_{2-}^{-}-\lambda\right) f_{3-}$ 和 $\left(f_{2-}^{-}-\lambda\right) f_{1-} f_{3-}$ 都属于 $H^{2}$, 其中 $f_{i-}=P_{-} f_{i}, i=1,2,3$.

\section{3 个 Hankel 算子乘积的另一个问题: 问题 1.2}

首先回顾问题 1.2. 设 $f, g, u \in H^{2}$, 则

$$
H_{\bar{f}} H_{\bar{u}}^{*} H_{\bar{g}}=H_{\bar{g}} H_{\bar{u}}^{*} H_{\bar{f}}
$$

成立的条件是什么?

本节的目标就是解决这个问题, 我们需要下面的引理.

引理 3.1 设 $f, g, u \in H^{2}$, 其中 $f$ 不是常数. 在 $H_{\bar{f}} H_{\bar{u}}^{*} H_{\bar{g}}=H_{\bar{g}} H_{\bar{u}}^{*} H_{\bar{f}}$ 成立的情况下, 如果存在 常数 $\lambda$, 使得 $(u-\lambda) \bar{f} \in H^{2}$, 则必有 $(u-\lambda) \bar{g} \in H^{2}$. 
证明 因为对于常数 $\lambda$, 任意有界 Hankel 算子 $H_{\phi}$ 满足 $H_{\phi}=H_{\phi+\lambda}$ 成立, 所以可以假设 $f(0)=$ $g(0)=0$. 根据已知条件 $H_{\bar{f}} H_{\bar{u}}^{*} H_{\bar{g}}=H_{\bar{g}} H_{\bar{u}}^{*} H_{\bar{f}}$ 可以推出

$$
H_{\bar{f}} H_{\bar{u}-\bar{\lambda}}^{*} H_{\bar{g}}=H_{\bar{g}} H_{\bar{u}-\bar{\lambda}}^{*} H_{\bar{f}} .
$$

因此,

$$
H_{\bar{f}} H_{\bar{u}-\bar{\lambda}}^{*} H_{\bar{g}} 1=H_{\bar{g}} H_{\bar{u}-\bar{\lambda}}^{*} H_{\bar{f}} 1
$$

直接计算上式可得

$$
\begin{aligned}
H_{\bar{f}} H_{\bar{u}-\bar{\lambda}}^{*} H_{\bar{g}} 1 & =H_{\bar{f}} H_{\bar{u}-\bar{\lambda}}^{*} \bar{g} \\
& =H_{\bar{f}} P(u-\lambda) \bar{g} \\
& =(I-P) \bar{f} P(u-\lambda) \bar{g} \\
& =\bar{f} P(u-\lambda) \bar{g}-P \bar{f} P(u-\lambda) \bar{g} \\
& =\bar{f} P(u-\lambda) \bar{g}-P(u-\lambda) \bar{f} \bar{g}
\end{aligned}
$$

和

$$
\begin{aligned}
H_{\bar{g}} H_{\bar{u}-\bar{\lambda}}^{*} H_{\bar{f}} 1 & =H_{\bar{g}} H_{\bar{u}-\bar{\lambda}}^{*} \bar{f} \\
& =H_{\bar{g}} P(u-\lambda) \bar{f} \\
& =(I-P) \bar{g}(u-\lambda) \bar{f},
\end{aligned}
$$

则

$$
\bar{f} P(u-\lambda) \bar{g}-P(u-\lambda) \bar{f} \bar{g}=(I-P)(u-\lambda) \bar{f} \bar{g} .
$$

整理上式可得

$$
\bar{f}[P(u-\lambda) \bar{g}-(u-\lambda) \bar{g}]=0 .
$$

根据假设 $f$ 不是常数, 可得

$$
P(u-\lambda) \bar{g}=(u-\lambda) \bar{g} \Rightarrow(u-\lambda) \bar{g} \in H^{2} .
$$

证毕.

下面是本节的主要结果.

定理 3.1 设 $f 、 g$ 和 $u$ 是属于 $H^{2}$ 的 3 个非常值函数, 则 $H_{\bar{f}} H_{\bar{u}}^{*} H_{\bar{g}}=H_{\bar{g}} H_{\bar{u}}^{*} H_{\bar{f}}$ 成立的充分必 要条件是下列条件之一成立:

(1) 存在常数 $\lambda$, 使得 $(u-\lambda) \bar{f}$ 和 $(u-\lambda) \bar{g}$ 都属于 $H^{2}$;

(2) $f$ 和 $g$ 的非平凡线性组合是常数.

证明 先证充分性. 如果 $f$ 和 $g$ 的非平凡线性组合为常数, 不失一般性, 可设 $f=a g+b$, 其中 $a$ 和 $b$ 为常数, 则 $H_{\bar{f}}=a H_{\bar{g}}$. 因此,

$$
H_{\bar{f}} H_{\bar{u}}^{*} H_{\bar{g}}=a H_{\bar{g}} H_{\bar{u}}^{*} H_{\bar{g}}, \quad H_{\bar{g}} H_{\bar{u}}^{*} H_{\bar{f}}=a H_{\bar{g}} H_{\bar{u}}^{*} H_{\bar{g}} .
$$


若存在常数 $\lambda$, 使得 $(u-\lambda) \bar{f}$ 和 $(u-\lambda) \bar{g}$ 都属于 $H^{2}$, 则对于 $z \in \mathbb{D}$, 有

$$
\begin{aligned}
H_{\bar{f}} H_{\bar{u}-\bar{\lambda}}^{*} H_{\bar{g}} K_{z} & =H_{\bar{f}} H_{\bar{u}-\bar{\lambda}}^{*}(\bar{g}-\bar{g}(z)) K_{z} \\
& =H_{\bar{f}} P(u-\lambda)(\bar{g}-\bar{g}(z)) K_{z} \\
& =P_{-} \bar{f}(u-\lambda)(\bar{g}-\bar{g}(z)) K_{z} \\
& =P_{-}(u-\lambda) \bar{f} \bar{g} K_{z}
\end{aligned}
$$

和

$$
\begin{aligned}
H_{\bar{g}} H_{\bar{u}-\bar{\lambda}}^{*} H_{\bar{f}} K_{z} & =H_{\bar{g}} P(u-\lambda)(\bar{f}-\bar{f}(z)) K_{z} \\
& =P_{-} \bar{g}(u-\lambda)(\bar{f}-\bar{f}(z)) K_{z} \\
& =P_{-}(u-\lambda) \bar{f} \bar{g} K_{z} .
\end{aligned}
$$

因此,

$$
H_{\bar{f}} H_{\bar{u}-\bar{\lambda}}^{*} H_{\bar{g}} K_{z}=H_{\bar{g}} H_{\bar{u}-\bar{\lambda}}^{*} H_{\bar{f}} K_{z} .
$$

由于 $\left\{K_{z}: z \in \mathbb{D}\right\}$ 的有限线性组合全体在 $H^{2}$ 中稠密, 因此,

$$
H_{\bar{f}} H_{\bar{u}-\bar{\lambda}}^{*} H_{\bar{g}}=H_{\bar{g}} H_{\bar{u}-\bar{\lambda}}^{*} H_{\bar{f}}
$$

和

$$
H_{\bar{f}} H_{\bar{u}}^{*} H_{\bar{g}}=H_{\bar{g}} H_{\bar{u}}^{*} H_{\bar{f}}
$$

成立.

下证必要性. 假设

$$
H_{\bar{f}} H_{\bar{u}}^{*} H_{\bar{g}}=H_{\bar{g}} H_{\bar{u}}^{*} H_{\bar{f}}
$$

成立. 由 (1.1) 可知, $H_{\bar{u}}^{*} H_{\bar{g}}=T_{u \bar{g}}-T_{u} T_{\bar{g}}$. 故

$$
\begin{aligned}
H_{\bar{f}} H_{\bar{u}}^{*} H_{\bar{g}} & =H_{\bar{f}}\left(T_{u \bar{g}}-T_{u} T_{\bar{g}}\right) \\
& =H_{\bar{f}} T_{u \bar{g}}-H_{\bar{f}} T_{u} T_{\bar{g}} \\
& =H_{\bar{f}} T_{u \bar{g}}-H_{u \bar{f}} T_{\bar{g}} .
\end{aligned}
$$

同理可得

$$
H_{\bar{g}} H_{\bar{u}}^{*} H_{\bar{f}}=H_{\bar{g}} T_{u \bar{f}}-H_{u \bar{g}} T_{\bar{f}} .
$$

这样 (3.1) 变为

$$
H_{\bar{f}} T_{u \bar{g}}-H_{u \bar{f}} T_{\bar{g}}=H_{\bar{g}} T_{u \bar{f}}-H_{u \bar{g}} T_{\bar{f}} .
$$

将 $I=T_{z} T_{\bar{z}}+1 \otimes 1$ 代入上式, 得

$$
H_{\bar{f}}\left(T_{z} T_{\bar{z}}+1 \otimes 1\right) T_{u \bar{g}}-H_{u \bar{f}}\left(T_{z} T_{\bar{z}}+1 \otimes 1\right) T_{\bar{g}}
$$




$$
=H_{\bar{g}}\left(T_{z} T_{\bar{z}}+1 \otimes 1\right) T_{u \bar{f}}-H_{u \bar{g}}\left(T_{z} T_{\bar{z}}+1 \otimes 1\right) T_{\bar{f}} .
$$

化简上式, 得

$$
\begin{aligned}
& H_{\bar{f}}(1 \otimes 1) T_{u \bar{g}}-H_{u \bar{f}}(1 \otimes 1) T_{\bar{g}}+S_{z}\left(H_{\bar{f}} T_{u \bar{z} \bar{g}}-H_{u \bar{f}} T_{\bar{z} \bar{g}}\right) \\
& \quad=H_{\bar{g}}(1 \otimes 1) T_{u \bar{f}}-H_{u \bar{g}}(1 \otimes 1) T_{\bar{f}}+S_{z}\left(H_{\bar{g}} T_{u \bar{z} \bar{f}}-H_{u \bar{g}} T_{\bar{z} \bar{f}}\right) .
\end{aligned}
$$

对上式两边同时复合算子 $T_{z}$, 得

$$
\begin{aligned}
& H_{\bar{f}}(1 \otimes 1) T_{z u \bar{g}}-H_{u \bar{f}}(1 \otimes 1) T_{z \bar{g}}+S_{z}\left(H_{\bar{f}} T_{u \bar{g}}-H_{u \bar{f}} T_{\bar{g}}\right) \\
& \quad=H_{\bar{g}}(1 \otimes 1) T_{z u \bar{f}}-H_{u \bar{g}}(1 \otimes 1) T_{z \bar{f}}+S_{z}\left(H_{\bar{g}} T_{u \bar{f}}-H_{u \bar{g}} T_{\bar{f}}\right) .
\end{aligned}
$$

因此,

$$
H_{\bar{f}}(1 \otimes 1) T_{z u \bar{g}}-H_{u \bar{f}}(1 \otimes 1) T_{z \bar{g}}=H_{\bar{g}}(1 \otimes 1) T_{z u \bar{f}}-H_{u \bar{g}}(1 \otimes 1) T_{z \bar{f}}
$$

再化简, 可得

$$
P_{-} \bar{f} \otimes P(\bar{z} \bar{u} g)-P_{-}(u \bar{f}) \otimes P(\bar{z} g)=P_{-} \bar{g} \otimes P(\bar{z} \bar{u} f)-P_{-}(u \bar{g}) \otimes P(\bar{z} f) .
$$

因为对于任意的常数 $\lambda$, 任何有界 Hankel 算子 $H_{\phi}$ 满足 $H_{\phi}=H_{\phi+\lambda}$. 若 $f$ 或 $g$ 为常数, 则定理 3.1(2) 自然成立. 为了方便讨论, 下面假设 $f(0)=g(0)=0$ 并且 $f$ 和 $g$ 都不为常数, 则

$$
P_{-} \bar{f}=\bar{f}, \quad P_{-} \bar{g}=\bar{g}, \quad P(\bar{z} g)=\bar{z} g \neq 0, \quad P(\bar{z} f)=\bar{z} f \neq 0 .
$$

这样 $(3.2)$ 就变成了

$$
\bar{f} \otimes P(\bar{z} \bar{u} g)-P_{-}(u \bar{f}) \otimes P(\bar{z} g)=\bar{g} \otimes P(\bar{z} \bar{u} f)-P_{-}(u \bar{g}) \otimes P(\bar{z} f) .
$$

接下来对以上的算子方程进行分类讨论.

情形 1 假设 $\bar{f} \otimes P(\bar{z} \bar{u} g) 、 P_{-}(u \bar{f}) \otimes P(\bar{z} g) 、 \bar{g} \otimes P(\bar{z} \bar{u} f)$ 和 $P_{-}(u \bar{g}) \otimes P(\bar{z} f)$ 中至少有一个为零 算子.

情形 1.1 设 $\bar{f} \otimes P(\bar{z} \bar{u} g)$ 是零算子, 由于 $f$ 不是常数, 则 $P(\bar{z} \bar{u} g)=0$, 这样可以推出 $\bar{z} \bar{u} g \in\left(H^{2}\right)^{\perp}$, 从而 $u \bar{g} \in H^{2}$. 由引理 3.1 知, $u \bar{f} \in H^{2}$, 这样定理 3.1(1) 成立.

情形 1.2 设 $P_{-}(u \bar{f}) \otimes P(\bar{z} g)$ 是零算子, 由于 $g$ 不是常数, 则

$$
P(\bar{z} g) \neq 0 \text { 推出 } \quad P_{-}(u \bar{f})=0 \text { 推出 } \quad u \bar{f} \in H^{2} .
$$

由引理 3.1 知 $u \bar{g} \in H^{2}$, 这样定理 3.1(1) 成立.

类似地, 当 $\bar{g} \otimes P(\bar{z} \bar{u} f)=0$ 或 $P_{-}(u \bar{g}) \otimes P(\bar{z} f)=0$ 时, 可以得到同样的结果 $u \bar{f}, u \bar{g} \in H^{2}$.

情形 2 假设 $\bar{f} \otimes P(\bar{z} \bar{u} g) 、 P_{-}(u \bar{f}) \otimes P(\bar{z} g) 、 \bar{g} \otimes P(\bar{z} \bar{u} f)$ 和 $P_{-}(u \bar{g}) \otimes P(\bar{z} f)$ 都不是零算子. 这样 就存在 $\lambda_{0} \in \mathbb{D}$, 使得

$$
P(\bar{z} \bar{u} g)\left(\lambda_{0}\right) \neq 0, \quad P(\bar{z} g)\left(\lambda_{0}\right) \neq 0, \quad P(\bar{z} \bar{u} f)\left(\lambda_{0}\right) \neq 0, \quad P(\bar{z} f)\left(\lambda_{0}\right) \neq 0 .
$$

将 (3.3) 两边作用在 $K_{\lambda_{0}}$ 上可得

$$
\left(\bar{f} \otimes P(\bar{z} \bar{u} g)-P_{-}(u \bar{f}) \otimes P(\bar{z} g)\right) K_{\lambda_{0}}=\left(\bar{g} \otimes P(\bar{z} \bar{u} f)-P_{-}(u \bar{g}) \otimes P(\bar{z} f)\right) K_{\lambda_{0}},
$$




$$
\overline{P(\bar{z} \bar{u} g)\left(\lambda_{0}\right)} \bar{f}-\overline{P(\bar{z} g)\left(\lambda_{0}\right)} P_{-}(u \bar{f})=\overline{P(\bar{z} \bar{u} f)\left(\lambda_{0}\right)} \bar{g}-\overline{P(\bar{z} f)\left(\lambda_{0}\right)} P_{-}(u \bar{g}) .
$$

因此, 存在非零常数 $a_{1} 、 a_{2}$ 和 $a_{3}$ 使得

$$
P_{-}(u \bar{g})=a_{1} \bar{g}+a_{2} P_{-}(u \bar{f})+a_{3} \bar{f} .
$$

将上式代入 (3.3), 可得

$$
\bar{g} \otimes\left(\overline{a_{1}} \bar{z} f-P(\bar{z} \bar{u} f)\right)+P_{-}(u \bar{f}) \otimes\left(\overline{a_{2}} \bar{z} f-\bar{z} g\right)+\bar{f} \otimes\left(\overline{a_{3}} \bar{z} f+P \bar{z} \bar{u} g\right)=0 .
$$

情形 2.1 假设 $\bar{g} \otimes\left(\overline{a_{1}} \bar{z} f-P(\bar{z} \bar{u} f)\right) 、 P_{-}(u \bar{f}) \otimes\left(\overline{a_{2}} \bar{z} f-\bar{z} g\right)$ 和 $\bar{f} \otimes\left(\overline{a_{3}} \bar{z} f+P \bar{z} \bar{u} g\right)$ 中至少有一个 是零算子, 根据假设, 即, $\left(\overline{a_{1}} \bar{z} f-P(\bar{z} \bar{u} f)\right) 、\left(\overline{a_{2}} \bar{z} f-\bar{z} g\right)$ 和 $\left(\overline{a_{3}} \bar{z} f+P \bar{z} \bar{u} g\right)$ 至少一个为 0 .

情况 2.1.1 如果 $\overline{a_{1}} \bar{z} f-P(\bar{z} \bar{u} f)=0$, 则 $\overline{a_{1}} \bar{z} f-\bar{z} \bar{u} f \in \overline{z H^{2}}$. 因此, $\overline{a_{1}} f-\bar{u} f \in \overline{H^{2}},\left(u-a_{1}\right) \bar{f} \in H^{2}$. 根据引理 3.1 知 $\left(u-a_{1}\right) \bar{g} \in H^{2}$, 这样定理 3.1(1) 成立.

情形 2.1.2 如果 $\overline{a_{2}} \bar{z} f-\bar{z} g=0$, 则 $\overline{a_{2}} f=g$, 定理 3.1(2) 成立.

情形 2.1.3 如果 $\overline{a_{3}} \bar{z} f+P(\bar{z} \bar{u} g)=0$, 则 $\bar{a}_{3} \bar{z} f+\bar{z} \bar{u} g \in\left(H^{2}\right)^{\perp}$. 这样 $\bar{a}_{3} f+\bar{u} g \in \overline{H^{2}}$. 从而,

$$
P_{-}(u \bar{g})=-a_{3} \bar{f}
$$

成立. 在这种情形的假设下, (3.5) 变为

$$
\bar{g} \otimes\left(\overline{a_{1}} \bar{z} f-P(\bar{z} \bar{u} f)\right)+P_{-}(u \bar{f}) \otimes\left(\overline{a_{2}} \bar{z} f-\bar{z} g\right)=0 .
$$

根据假设, 我们只需要考虑 $\bar{g} 、\left(\overline{a_{1}} \bar{z} f-P(\bar{z} \bar{u} f)\right) 、 P_{-}(u \bar{f})$ 和 $\left(\overline{a_{2}} \bar{z} f-\bar{z} g\right)$ 都不是零的情形. 因此存 在非零常数 $a_{4}$ 使得

$$
P_{-}(u \bar{f})=a_{4} \bar{g}
$$

将 (3.6) 和 (3.7) 代入 (3.4), 可得

$$
-2 a_{3} \bar{f}=\left(a_{1}+a_{2} a_{4}\right) \bar{g}
$$

因此定理 3.1(2) 成立.

情形 2.2 假设 $\bar{g} \otimes\left(\overline{a_{1}} \bar{z} f-P(\bar{z} \bar{u} f)\right) 、 P_{-}(u \bar{f}) \otimes\left(\overline{a_{2}} \bar{z} f-\bar{z} g\right)$ 和 $\bar{f} \otimes\left(\overline{a_{3}} \bar{z} f+P \bar{z} \bar{u} g\right)$ 都不是零算子, 则存在常数 $\lambda \in \mathbb{D}$, 使得 $\left(\overline{a_{1}} \bar{z} f-P(\bar{z} \bar{u} f)\right)(\lambda) 、\left(\overline{a_{2}} \bar{z} f-\bar{z} g\right)(\lambda)$ 和 $\left(\overline{a_{3}} \bar{z} f+P \bar{z} \bar{u} g\right)(\lambda)$ 都不是 0, 将 $(3.5)$ 作用在 $K_{\lambda}$ 可得

$$
\begin{aligned}
(\bar{g} & \left.\otimes\left(\overline{a_{1}} \bar{z} f-P(\bar{z} \bar{u} f)\right)+P_{-}(u \bar{f}) \otimes\left(\overline{a_{2}} \bar{z} f-\bar{z} g\right)+\bar{f} \otimes\left(\overline{a_{3}} \bar{z} f+P \bar{z} \bar{u} g\right)\right) K_{\lambda} \\
& =\left\langle K_{\lambda}, \overline{a_{1}} \bar{z} f-P(\bar{z} \bar{u} f)\right\rangle \bar{g}+\left\langle K_{\lambda}, \overline{a_{2}} \bar{z} f-\bar{z} g\right\rangle P_{-}(\bar{f} u)+\left\langle K_{\lambda}, \overline{a_{3}} \bar{z} f+P \bar{z} \bar{u} g\right\rangle \bar{f} \\
& =\left(\overline{a_{1}} \bar{z} f-P(\bar{z} \bar{u} f)\right)(\lambda) \bar{g}+\left(\overline{a_{2}} \bar{z} f-\bar{z} g\right)(\lambda) P_{-}(\bar{f} u)+\left(\overline{a_{3}} \bar{z} f+P \bar{z} \bar{u} g\right)(\lambda) \bar{f} \\
& =0 .
\end{aligned}
$$

因此存在非零常数 $b_{1}$ 和 $b_{2}$ 使得

$$
P_{-}(\bar{f} u)=b_{1} \bar{g}+b_{2} \bar{f} .
$$


将 (3.8) 代入 (3.5), 可得

$$
\bar{g} \otimes\left(\overline{b_{1}}\left(\overline{a_{2}} \bar{z} f-\bar{z} g\right)+\overline{a_{1}} \bar{z} f-P(\bar{z} \bar{u} f)\right)=-\bar{f} \otimes\left(\overline{b_{2}}\left(\overline{a_{2}} \bar{z} f-\bar{z} g\right)+\overline{a_{3}} \bar{z} f+P \bar{z} \bar{u} g\right) .
$$

情形 2.2.1 如果 $\left(\overline{b_{1}}\left(\overline{a_{2}} \bar{z} f-\bar{z} g\right)+\overline{a_{1}} \bar{z} f-P(\bar{z} \bar{u} f)\right)$ 和 $\left(\overline{b_{2}}\left(\overline{a_{2}} \bar{z} f-\bar{z} g\right)+\overline{a_{3}} \bar{z} f+P \bar{z} \bar{u} g\right)$ 都不是 0 , 则 $f$ 是 $g$ 的常数倍.

情形 2.2.2 由假设知, $f$ 和 $g$ 都不是 0 . 令 $\overline{b_{1}}\left(\overline{a_{2}} \bar{z} f-\bar{z} g\right)+\overline{a_{1}} \bar{z} f-P(\bar{z} \bar{u} f)=0$, 则

$$
\overline{b_{2}}\left(\overline{a_{2}} \bar{z} f-\bar{z} g\right)+\overline{a_{3}} \bar{z} f+P \bar{z} \bar{u} g=0 .
$$

因此,

$$
0=\overline{b_{1}}\left(\overline{a_{2}} \bar{z} f-\bar{z} g\right)+\overline{a_{1}} \bar{z} f-P(\bar{z} \bar{u} f)=\left(\overline{a_{2}} \overline{b_{1}}+\overline{a_{1}}\right) \bar{z} f-\overline{b_{1}} \bar{z} g-P(\bar{z} \bar{u} f)
$$

则

$$
\overline{\left(a_{2} b_{1}+a_{1}\right)} f-\bar{b}_{1} g-\bar{u} f \in \overline{H^{2}} .
$$

于是,

$$
0=\left(a_{2} b_{1}+a_{1}\right) \bar{f}-b_{1} \bar{g}-P_{-}(\bar{f} u) .
$$

结合 (3.8) 和 (3.10) 知, 定理 3.1(2) 成立. 类似地, 考虑 $\overline{b_{2}}\left(\overline{a_{2}} \bar{z} f-\bar{z} g\right)+\overline{a_{3}} \bar{z} f+P \bar{z} \bar{u} g=0$. 定理证毕.

例 3.1 如果 $u$ 是内函数, $f, g \in H^{2} \cap u \overline{H^{2}}$, 则

$$
H_{\bar{f}} H_{\bar{u}}^{*} H_{\bar{g}}=H_{\bar{g}} H_{\bar{u}}^{*} H_{\bar{f}} .
$$

证明 因为 $f, g \in H^{2} \cap u \overline{H^{2}}$, 所以存在 $f_{1}, g_{1} \in H^{2}$ 使得

$$
f=u \bar{f}_{1}, \quad g=u \bar{g}_{1} .
$$

这样, $u \bar{f}=u\left(\bar{u} f_{1}\right)=f_{1} \in H^{2}$ 和 $u \bar{g}=u\left(\bar{u} g_{1}\right)=g_{1} \in H^{2}$. 由定理 3.1 可得 $H_{\bar{f}} H_{\bar{u}}^{*} H_{\bar{g}}=H_{\bar{g}} H_{\bar{u}}^{*} H_{\bar{f}}$.

致谢 衰心感谢审稿人的宝贵意见.

\section{参考文献}

1 Xia D, Zheng D. Products of Hankel operators. Integral Equations Operator Theory, 1997, 29: 339-363

2 Sarason D. Algebraic properties of truncated Toeplitz operators. Oper Matrices, 2007, 1: 491-526

3 Baranov A, Chalendar I, Fricain E, et al. Bounded symbols and reproducing Kernel thesis for truncated Toeplitz operators. J Funct Anal, 2010, 259: 2673-2701

4 Ma P, Zheng D. Compact truncated Toeplitz operators. J Funct Anal, 2016, 270: 4256-4279

5 Ding X, Sang Y. Dual truncated Toeplitz operators. J Math Anal Appl, 2018, 461: 929-946

6 Yoshino T. The conditions that the product of Hankel operators is also a Hankel operator. Arch Math (Basel), 1999, 73: $146-153$

7 Brown A, Halmos P. Algebraic properties of Toeplitz operators. J Reine Angew Math, 1964, 1964: 89-102

8 Ahern P. On the range of the Berezin transform. J Funct Anal, 2004, 215: 206-216

9 Ahern P, Cuučković Ž. A theorem of Brown-Halmos type for Bergman space Toeplitz operators. J Funct Anal, 2001, 187: $200-210$ 


\section{Two questions on products of Hankel operators}

\section{Xuanhao Ding \& Yuanqi Sang}

Abstract Suppose $f, u$ and $g$ are in the Hardy space of the unit circle $H^{2}$, and $h$ is a square integrable function on the unit circle. $H_{\bar{f}}, H_{\bar{u}}, H_{\bar{g}}$ and $H_{h}$ are Hankel operators which take the Hardy space of the unit circle $H^{2}$ into the orthogonal complement of the Hardy space $\left(H^{2}\right)^{\perp}$. We obtain necessary and sufficient conditions for $H_{\bar{f}} H_{\bar{u}}^{*} H_{\bar{g}}=H_{h}$ and $H_{\bar{f}} H_{\bar{u}}^{*} H_{\bar{g}}=H_{\bar{g}} H_{\bar{u}}^{*} H_{\bar{f}}$, respectively.

Keywords Hankel operator, Hardy space, product

MSC(2020) 47B35

doi: 10.1360/N012019-00017 